\title{
The Role of CT Angiography in Upper GI Bleeding
}

\author{
R Darracott MBChB, FY2, UA Sheikh MRCP(UK), N Nik-Hussin MRCS, FRCR
}

\section{BACKGROUND}

50 to 150 per 100,000 cases of upper gastrointestinal bleeding (UGIB) are seen annually in the United Kingdom (UK). There is an increased risk of mortality in cases where blood loss is rapid enough to cause hypovolemic shock. ${ }^{1}$

Acute UGIB, defined as loss of blood proximal to the ligament of Treitz, is a medical emergency. This bleeding is usually characterised by occurrence of fresh haematemesis, coffee-ground emesis or melaena (dark tarry stools due to the effect of digestive enzymes on blood). The passage of fresh red blood per rectum, termed haematochezia, may also occur in rare cases where the rate and volume of blood loss is high and blood transits rapidly enough that blood is passed per rectum unaltered. ${ }^{2}$

Diagnosis of the cause of UGIB can sometimes prove to be elusive. The recommended primary diagnostic option of upper gastrointestinal endoscopy reveals the diagnosis in $95 \%$ of cases, ${ }^{3}$ in the remainder of cases a computed tomography (CT) angiogram has been shown to detect bleeding sites as low as $0.5 \mathrm{~mL} / \mathrm{min}$. Its sensitivity in UGIB is up to $98 \%$ with a specificity of $100 \% .^{4}$

\section{CASE PRESENTATION}

An 83-year-old gentleman presented to the emergency department complaining of several days of progressively worsening breathlessness and lethargy. On further questioning he mentioned noticing that his stools had become darker over the past 24 hours, but denied vomiting. He stated he had a few weeks history of haematuria in addition to his symptoms, but this had been investigated and an irregular prostate growth was discovered.

His past medical history comprised of gout, type 2 diabetes, atrial fibrillation and a metallic aortic valve replacement for which he was anticoagulated with warfarin. Of note, he had also recently undergone radiotherapy for recurrence of colon cancer. On examination the patient was noted to be pale, and subsequent digital rectal examination revealed the presence of melaena.

\section{INVESTIGATIONS}

Initial laboratory investigations were as follows:

\begin{tabular}{|l|l|l|l|}
\hline Hb & $66(\downarrow)$ & WCC & $14.5(\uparrow)$ \\
\cline { 1 - 2 } INR & $4.9(\uparrow)$ & Neutrophils & $12.2(\uparrow)$ \\
\hline Urea & $54.8(\uparrow)$ & CRP & $<5$ \\
\cline { 1 - 2 } Creatinine & $161(\uparrow)$ & \multicolumn{1}{|}{} \\
\cline { 1 - 2 } eGFR & $39(\downarrow)$ & \multicolumn{1}{|c}{} \\
\cline { 1 - 2 } & &
\end{tabular}

\section{INVESTIGATION AND TREATMENT}

This patient was transfused a total of four units of red blood cells (RBC), two in the emergency department and two in the acute medical unit, and his warfarin was reversed with $2 \mathrm{mg}$ Vitamin K. Oesophagogastroduodenoscopy (OGD) was performed, which was unable to reveal an active site of bleeding so it was assumed this had self-terminated. On this basis anticoagulation was continued with treatmentdose enoxaparin and discharge provisionally planned for a few days' time. A few hours later, he passed a further large black tarry stool and was transfused two units of blood overnight. CT angiogram was performed the next morning which showed "a small blush of contrast extravasation in the second part of the duodenum on arterial phase (see Fig. 2)". OGD was repeated, revealing bleeding $12 \mathrm{~mm}$ polypoid lesion at D1/D2 which was successfully treated with argon plasma therapy. After a few days of inpatient monitoring no further bleeding recurred and the patient was discharged.

\section{Aetiology of Acute Upper GI Bleed}

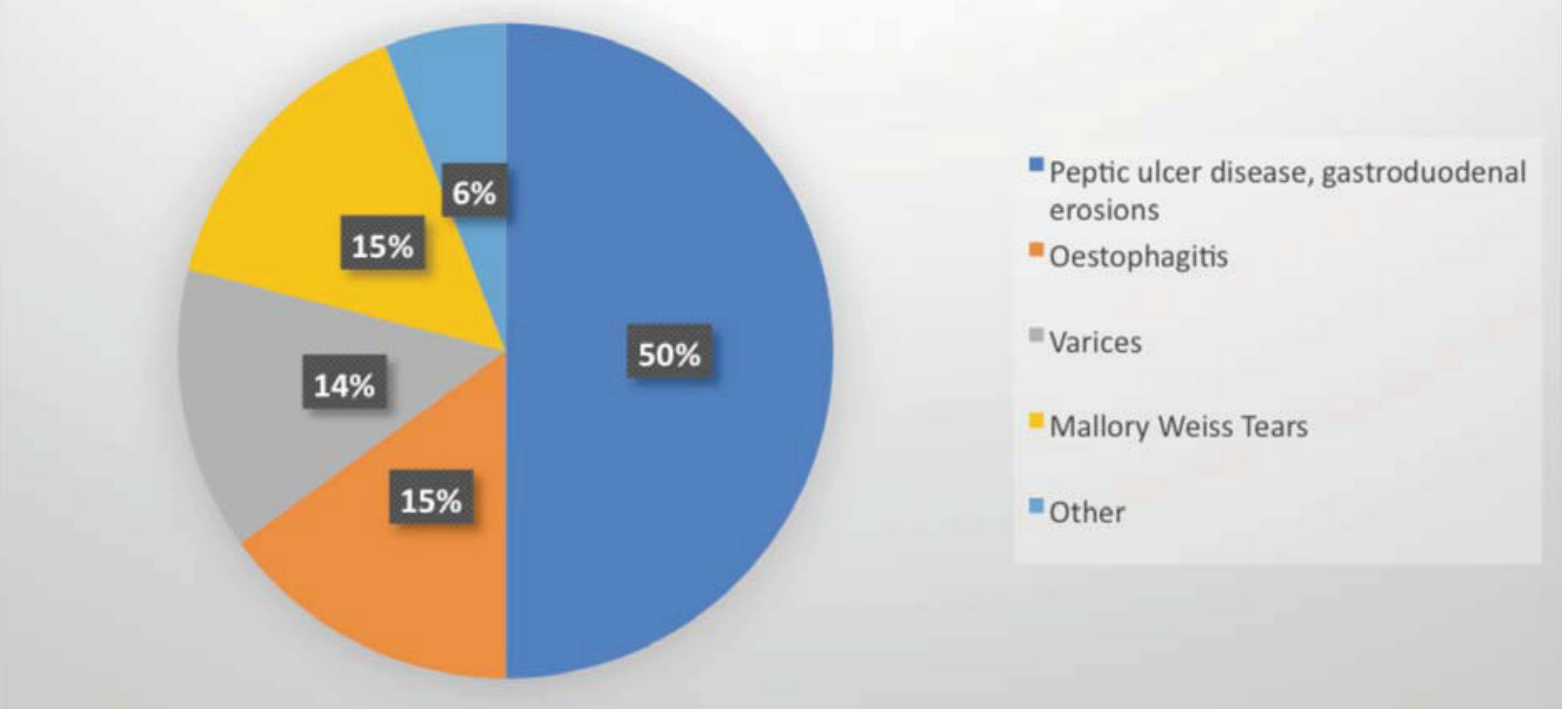

Figure 1: Etiology of Aetiological Factors of UGIB.? 


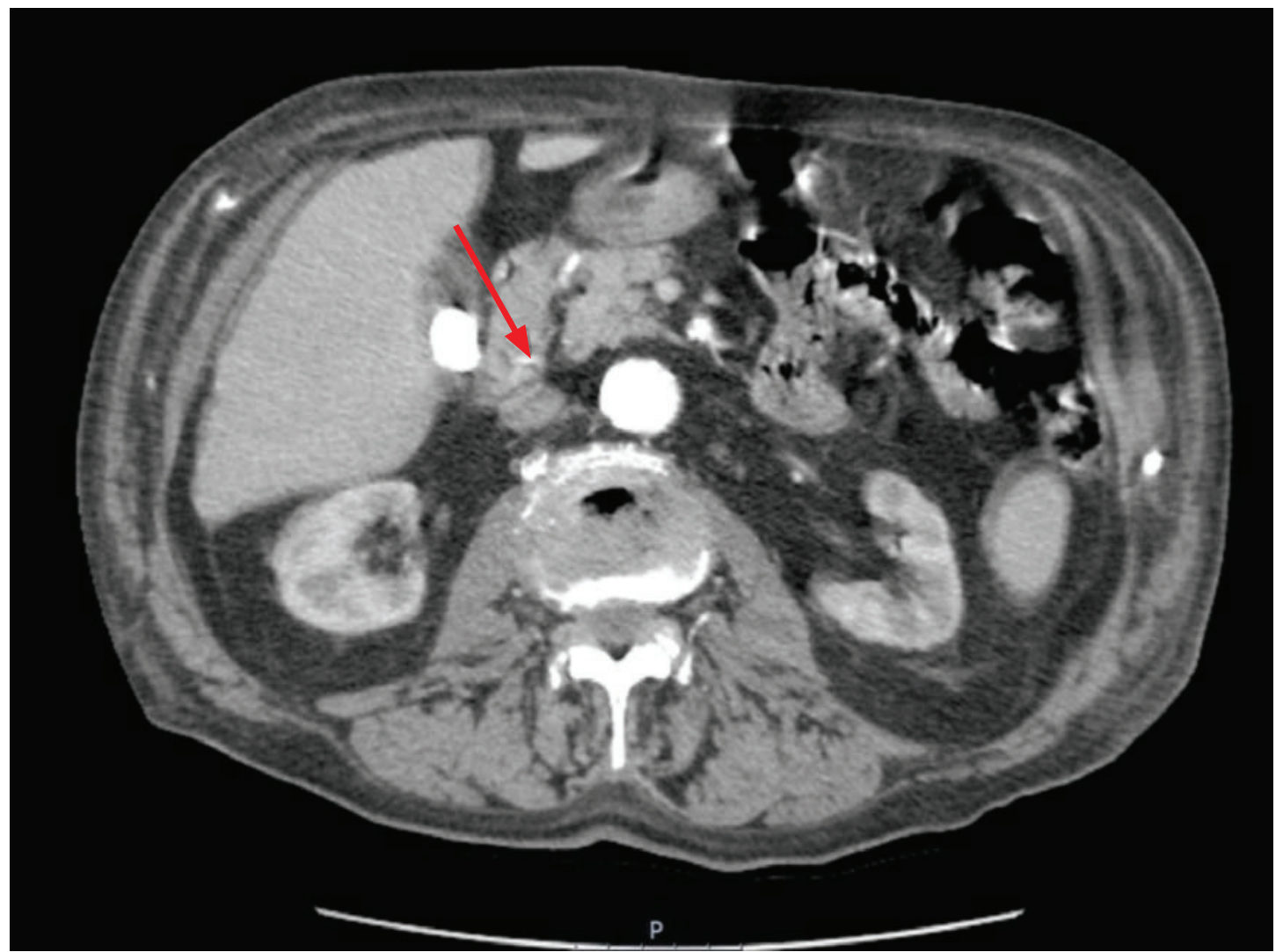

Figure 2: Axial slice of CT angiogram showing extravasation of contrast from bleeding site in duodenum (arrow).

\section{LEARNING POINTS}

- OGD is first line in the investigation and management of UGIB. For patients who rebleed or are at high risk of rebleeding, NICE recommends repeating OGD. ${ }^{5}$

- Transcatheter arterial embolisation (TAE) is recommended above repeat endoscopy in cases where the patient is haemodynamically unstable. If interventional radiology is not available promptly, surgery is the next therapeutic option. ${ }^{5}$

- Despite NICE recommendations, the 2015 NCEPOD audit shows just $27 \%$ of hospitals could provide access to TAE 24-hours a day. In practice, hospitals without acute access to interventional radiology, could utilise CT angiography to provide useful information, such as bleeding site, to guide the subsequent management of high-risk patients. ${ }^{6}$

\section{REFERENCES}

1. NICE. Acute upper gastrointestinal bleeding: costing report. Implementing NICE guidance. June 2012. Available at: https://www.nice.org.uk/guidance/ cg141/ (last accessed 6.4.17).

2. Wilcox CM, Alexander LN, Cotsonis G. A prospective characterization of upper gastrointestinal hemorrhage presenting with hematochezia. Am J Gastroenterol. 1997;92:231-235.

3. Millward, S. ACR Appropriateness Criteria ${ }^{\circledR}$ on treatment of acute nonvariceal gastrointestinal tract bleeding. Journal of the American College of Radiology 2008;5(4):550-554.
4. Wortman JR, Landman W, Fulwadhva UP, Viscomi SG, Sodickson AD. CT angiography for acute gastrointestinal bleeding: what the radiologist needs to know. Br J Radiol. 2017, Jul;90:(1075):20170076.

5. NICE (2016). Acute upper gastrointestinal bleeding in over 16s: management. Available at: https:// www.nice.org.uk/guidance/CG141/chapter/1Guidance\#timing-of-endoscopy (accessed 18.2.19).

6. McPherson S, Sinclair M, Smith N, Kelly K, Ellis D, Mason M. NCEPOD - Gastrointestinal Haemorrhage: Time to Get Control? (2015). Available at: https://www.ncepod.org.uk/2015gih. html (accessed 18.2.19).

7. Rockall TA, Logan RF, Devlin HB et al. Incidence of and mortality from acute upper gastrointestinal haemorrhage in the United Kingdom. Steering Committee and members of the National Audit of Acute Upper Gastrointestinal Haemorrhage. BMJ 1995;311:222-226.

8. Tarasconi A, Baiocchi G, Pattonieri V, Perrone G, Abongwa H, Molfino S. et al. Transcatheter arterial embolization versus surgery for refractory nonvariceal upper gastrointestinal bleeding: a metaanalysis. World Journal of Emergency Surgery 2019;14(1). Available at: https://wjes.biomedcentral. com/articles/10.1186/s13017-019-0223-8 (accessed 18.2.19).

Correspondence to: rebecca.darracott@mbht.nhs.uk 